\title{
Safety and Feasibility of Neurofeedback Training (NFB) During Sleep in Uncooperative Child with Autism: Case Report
}

\author{
Keren Avirame ${ }^{1 *}$, Limor Nuss ${ }^{2}$, and Doron Todder ${ }^{3}$ \\ ${ }^{1}$ Neuroclinic Health Center, Ramat Gan, Israel \\ ${ }^{2}$ Beersheva Mental Health Center, Beersheva, Israel \\ ${ }^{3}$ Zlotovsky Center for Neuroscience, Ben-Gurion University, Beersheva, Israel
}

\begin{abstract}
Purpose: Neurofeedback (NFB) training has demonstrated significant potential in achieving symptoms reduction in children with Autism Spectrum Disorder (ASD). However, children with low-functioning ASD are often uncooperative with the treatment. To evaluate whether NFB can eventually be administrated during sleep, a safety and feasibility pilot study was performed. Methods: A 9-year-old male patient diagnosed with ASD underwent NFB training for $30 \mathrm{~min}$ twice a week. This was operated at home during sleep by the parents. The NFB protocol aimed at increasing sensorimotor rhythm (SMR) while simultaneously decreasing theta activity over the sensorimotor strip. Results: NFB during sleep was feasible and did not yield adverse side effects. Parents reported improved behavioral and emotional symptoms and enhanced language development following NFB training. Subsequently, the patient could participate in regular sessions of NFB in wakefulness. Conclusion: Overall, parental reports suggest that applying NFB during sleep in low-functioning ASD is feasible and might offer promising therapeutic avenues.
\end{abstract}

Keywords: sleep; neurofeedback; Autism; sensorimotor rhythm

Citation: Avirame, K., Nuss, L., \& Todder, D. (2016). Safety and feasibility of neurofeedback training (NFB) during sleep in uncooperative child with Autism: case report. NeuroRegulation, 3(3), 135-139. http://dx.doi.org/10.15540/nr.3.3.135

*Address correspondence to: $\mathrm{Dr}$. Keren Avirame, Neuroclinic

Health Center, Menachem Begin Road 7, Ramat Gan, Israel 52681.

Email: research@neuroclinic.co.il

Copyright: (c) 2016. Avirame et al. This is an Open Access article distributed under the terms of the Creative Commons Attribution License (CC-BY).

\section{Edited by:}

Rex L. Cannon, PhD, Neurogifted, Boynton Beach, Florida, USA

Reviewed by:

Rex L. Cannon, PhD, Neurogifted, Boynton Beach, Florida, USA Randall Lyle, PhD, Mount Mercy University, Cedar Rapids, lowa, USA

\section{Introduction}

Neurofeedback is a specific form of biofeedback aiming at changing EEG oscillations through operant conditioning (Sterman, 2000). Normally, NFB protocols are determined on the basis of spectral parameters of spontaneous EEG in resting-state conditions. This EEG signal is processed and compared to database of normal subjects in order to reveal a profile of abnormalities (Coben \& Padolsky, 2007). Changes in EEG patterns as a result of NFB have been associated with regulation of cerebral blood flow, metabolism, and neurotransmitter function (Lubar, 1997). On the behavioral level, changes in cognitive and emotional functions have been documented in healthy individuals (Gruzelier,
2014), as well as in neuropsychiatric patients (e.g., Hammond, 2005; Sterman \& Egner, 2006).

The relationship between NFB training and sleep can be viewed as two-way counter paths. From the beginning, NFB has been frequently used to treat sleep disturbances since facilitating specific oscillations during wakefulness selectively enhances similar brain patterns during subsequent sleep period (Hoedlmoser et al., 2008). Particularly, it was proposed that improvement of physiological regulation of sensorimotor rhythm (i.e., low-beta frequency band $12-15 \mathrm{~Hz}$ ) stabilizes sleep state through optimization of thalamic-cortical circuits (Sterman, 2000). Sensorimotor rhythm (SMR) is also thought to be related to the intrinsic function of the reticular activating system (RAS), which, among 
other things, is connected to the regulation of wakefulness and sleep-wake transitions (Sterman \& Bowersox, 1981).

The parallel, yet opposite, approach is to modulate brain activity during sleep in order to alter long-term behavior at wakefulness. According to Tononi and Cirelli (2006), sleep promotes homeostasis by reducing the strength of synaptic connections to a level that is energetically sustainable and thus primes new learning. Arzi and colleagues (2014) recently reported that olfactory aversive conditioning during sleep significantly reduced cigarette-smoking behavior for several days. Moreover, methods of neuromodulation such as transcranial direct current stimulation (tDCS) were found to enhance retention of learned material when applied during sleep, pointing to the possibility of "night-treatment" for memory enhancement (Marshall, Mölle, Hallschmid, \& Born, 2004). Together, these studies confirm that sleep might be particularly beneficial for learning (Dudai, 2012).

ASD is a pervasive developmental disorder including a variety of cognitive, sensory, and social deficits such as impaired communication skills and sociability, as well as restricted and repeated behaviors (Hill \& Frith, 2003). NFB training in ASD with theta suppression and SMR enhancement were reported to induce positive short- and long-term effects on executive functions, social interaction, and communication skills (Kouijzer, de Moor, Gerrits, Buitelaar, \& van Schie, 2009; Kouijzer, de Moor, Gerrits, Congedo, \& van Schie, 2009). Enhancement of SMR is reported to improve response inhibition and promote synchronization by regulating the sensorimotor system (Gruzelier \& Egner, 2005), while suppression of theta is reported to promote more flexible functioning of the brain by activating the default mode network (Kouijzer, de Moor, Gerrits, Buitelaar, \& van Schie, 2009).

Although a considerable number of children with ASD cooperate with NFB training, some with more pronounced symptoms of restlessness, hypersensitivity, and aggression cannot receive treatments due the severity of their symptoms. This is unfortunate in the face of a growing number of studies revealing the benefits of NFB in children and adults diagnosed with ASD (Holtmann et al., 2011). Given it is important to accommodate NFB to a wide range of patients, treatment during sleep could act as a possible solution for lack of cooperativeness. Similar to many medical interventions, NFB can induce adverse effects when it is commonly implemented in wakefulness (Rogel et al., 2015); here, we were specifically concerned about inducing sleep disturbance as a result of the night-treatment.

Thus, the purpose of this pilot was to evaluate whether NFB training during sleep is safe and feasible and whether it could be behaviorally beneficial for children with ASD. Here we applied NFB training using a reward sound during sleep. The training was set to up-regulate SMR $(11-14 \mathrm{~Hz})$ and down-regulate theta $(4-7 \mathrm{~Hz})$ located across the sensorimotor strip. This protocol was chosen since it has been shown to regulate altered neuronal excitability (Sterman, 2000). Of note, uncooperativeness observed in this kind of patient often dictates prototypic use of research-based protocols, as EEG could not be properly recorded and analyzed. Along the treatment, the parents needed to evaluate (a) adverse side effects, specifically, in regard to sleep, (b) feasibility, that is, how easy it is to operate the treatment during sleep, and (c) efficacy, whether they observed reduction in ASD symptoms.

\section{Neurofeedback}

NFB was administered at central electrode sites (C4, $\mathrm{Cz}$, and $\mathrm{C} 3$ ) with BrainMaster 2EB system with the 3.7 $i$ software (BrainMaster Technologies, Inc., Bedford, $\mathrm{OH}$ ). Patients received feedback in a form of auditory sound on their real-time EEG signal, while sleeping. The volume of the auditory sound was adjusted by the parents to ensure that sleep is not interrupted. EEG data were obtained from the active electrode placed on the scalp at the location(s) of interest. The active electrode was stabilized on the desired location with Ten20 conductive electrode paste. Both the reference and the ground electrodes were linked to the ipsilateral earlobe. Sampling rate was $256 \mathrm{~Hz}$. Reward threshold levels were automatically adjusted based on the digitally filtered real-time EEG signal every $180 \mathrm{~s}$. The percent of reward was $70 \%$ of the time for the up-regulation of SMR and $70 \%$ of the downregulation of theta (inhibition level of $30 \%$ ends with $70 \%$ rewards). The time period for getting a reward was fixed to $250 \mathrm{~ms}$. Therefore, the patient received rewards for maximum $49 \%$ of the time. The treatment was operated by the parents following the installation of the BrainMaster software on their private laptop. Finally, the parents were trained to attach the electrodes and to use the system through the home training configuration option. 


\section{Procedure}

This is a retrospective summary of treatment performed on one patient. NFB sessions were administered twice per week for $30 \mathrm{~min}$ approximately 30 minutes after the child fell asleep. The treatment was performed using a laptop at the child bedroom. The parent had to ensure that the treatment was carried on during sleep. In case the child woke up during the session or the child repeatedly moved, the treatment was interrupted. The volume of the reward sound was modulated by the parent to maintain sleep. All subjective reports were sent by the parents to the therapist in charge via emails. These reports were filled out at the end of each session, indicating behavioral changes which took place in between the two last sessions. Due to technical problems related to the computer used for home training, EEG data during the training was not properly saved and therefore could not be presented in this case report. The range of SMR and theta bands was minimally modified when needed, as explained below.

\section{SV}

$\mathrm{SV}$ is a right-handed, 9-year-old male diagnosed with ASD. He attends a special needs school and follows speech therapy due to severe communication disability. His main symptoms included: speech limited to single syllables, lack of eye contact, restlessness, and aggression. After we failed to perform 19-channel EEG recording and to do regular NFB in wakefulness, the parents were offered to try NFB during sleep. The parents were given detailed explanation about the lack of evidence to support the effectiveness of such procedure. Following their decision, they signed an informed consent form for this treatment.

The treatment started with 30 min of NFB training for SMR $(12-15 \mathrm{~Hz})$ uptraining and theta $(4-7 \mathrm{~Hz})$ downtraining over $\mathrm{C} 4$. This protocol was delivered for eight sessions. Already after the second session, SV was more calm and cooperative and made more efforts to name objects. This improvement was also noticeable during his speech therapy. The first protocol was followed by $30 \mathrm{~min}$ of NFB training for SMR $(11-14 \mathrm{~Hz})$ uptraining and theta $(4-7 \mathrm{~Hz})$ downtraining over $\mathrm{C} 4$ for another 24 sessions. The change in SMR frequency range resulted from increasing boisterousness which disappeared shortly after the change took place.

From the $15^{\text {th }}$ session, the patient showed increased attention, environmental awareness, and responsiveness to others. He also started to say words of three-syllable length, pronounce words more clearly, mumble non-words, produce sounds, and simulate music tunes. For the rest of the sessions, the electrode was located on $\mathrm{C} 3$ in order to increase left-hemisphere functions such as language development. Consequently, the patient showed improved understanding and was more successful in accomplishing complex tasks and following instructions. Overall, SV received 60 sessions during sleep. No side effects were reported during treatment period. After 60 sessions his behavior improved to the point that he could participate in regular sessions of NFB in wakefulness.

\section{Discussion}

Here we applied for the first time NFB treatment during sleep in a patient with low-functioning ASD who was uncooperative during wakefulness due to his symptoms. We applied a conventional protocol focusing on SMR and theta activity without prior examination of spontaneous EEG oscillations because the patient was uncooperative with the examination. Of note, the use of EEG norms during sleep is not applicable since all EEG norms were analyzed and measured during wakefulness and not during sleep. We found that home training during sleep is similar to supervised training during wakefulness in the clinic. In other words, the change in settings doesn't necessarily undermine the efficacy of the treatment. However, it is important to optimize the use of home application of NFB system to the point that training sessions could be stored and then analyzed, not only for ensuring the quality of the treatment, but also for plotting a learning curve to examine the changes in the trained brain waves.

In regard to safety concerns, the treatment did not cause sleep disturbances in this patient. In fact, the parents did not observe changes in sleep patterns (e.g., increased movement, waking up, speaking, dreaming) and wakefulness (e.g., tiredness, daydreaming, alertness, etc.) as a result of the treatment. Other adverse side effects were not reported. Along the treatment, the parents observed a variety of benefits ranging from improved speech and comprehension to increased attention, as well as decrease in uncooperativeness, restlessness, and aggression. In other words, regulating SMR and theta during sleep led to positive long-term effects in wakefulness. Importantly, the patient improved to the point of being able to cooperate with the regular treatment procedure, which involved 
watching a film for $30 \mathrm{~min}$ with electrodes on the ears and scalp, while keeping focus on the screen and minimal movement.

The possible mechanisms of the treatment-induced benefits are open for discussion. One explanation might posit that the observed effects result from improving sleep patterns or regulating epileptic activity. Indeed, the association between ASD and epilepsy was found to be higher in low-functioning ASD (Tuchman, 2000); higher predisposition to chronic disturbances in sleep-wake cycle was found in low-functioning ASD in dependence of the degree and severity of their cognitive impairment (Sajith \& Clarke, 2007). Obviously, there is the possibility that the observed improvements stem from treating ADHD like symptoms, which are in high co-morbidity in ASD (Holtmann, Bölte, \& Poustka, 2007). In other words, improved daytime behavior in this patient might be mediated by optimizing sleep patterns, improving seizure activity, and reducing hyperactivity.

Although many questions regarding the underlying mechanisms of these reported improvements remain unclear, it is first necessary to validate the current pilot through controlled trial on a group of children with ASD. Taking into account that researchers have identified a number of Autism subtype EEG patterns (Coben, Linden, \& Myers, 2010), other protocols should also be considered. At this point, we only attempted to test the feasibility and safety in applying NFB training during sleep. Given these treatment effects were reported by the parents of the patients, future research should target specific symptoms that can be evaluated by standardized measurements.

To conclude, NFB training is a promising technique for normalizing and optimizing brain activity. Due to the automaticity of operant-conditioning NFB is also applicable in states of reduced consciousness such as state of unresponsive wakefulness (Keller \& Garbacenkaite, 2015) as well as in coma (Ayers, 1999). Here we applied it successfully for the first time during sleep in line with multiple reports on its beneficial effects on children with ASD. Despite the various limitations that this preliminary work entails, the sleep training was found to be safe and feasible, and enabled to later continue with regular training in wakefulness.

\section{Author Notes:}

This study was not funded.

The authors have no conflicts of interest to report.
The study was performed in accordance with the 1964 Helsinki declaration.

Informed consent was obtained from the patient included in the study.

\section{References}

2EB system [Apparatus]. Bedford, $\mathrm{OH}$ : BrainMaster Technologies, Inc.

Arzi, A., Holtzman, Y., Samnon, P., Eshel, N., Harel, E., \& Sobel, N. (2014). Olfactory aversive conditioning during sleep reduces cigarette-smoking behavior. The Journal of Neuroscience, 34(46), 15382-15393. http://dx.doi.org /10.1523/JNEUROSCI.2291-14.2014

Ayers, M. E. (1999). Assessing and treating open head trauma, coma, and stroke using real-time digital EEG neurofeedback. Introduction to Quantitative EEG and Neurofeedback, 203222. http://dx.doi.org/10.1016/B978-012243790-8/50010-9

BrainMaster (Version 3.7i) [Computer software]. Bedford, $\mathrm{OH}$ : BrainMaster Technologies, Inc. Retrieved from: http://www.brainmaster.com/software/3.0/BMr30Setup3.7i.ex

Coben, R., Linden, M., \& Myers, T. E. (2010). Neurofeedback for autistic spectrum disorder: A review of the literature. Applied Psychophysiology and Biofeedback, 35(1), 83-105. http://dx.doi.org/10.1007/s10484-009-9117-y

Coben, R., \& Padolsky, I. (2007). Assessment-guided neurofeedback for autistic spectrum disorder. Journal of Neurotherapy, 11(1), 5-23. http://dx.doi.org/10.1300 IJ184v11n01_02

Dudai, Y. (2012). The restless engram: Consolidations never end. Annual Review of Neuroscience, 35, 227-247. http://dx.doi.org/10.1146/annurev-neuro-062111-150500

Gruzelier, J., \& Egner, T. (2005). Critical validation studies of neurofeedback. Child and Adolescent Psychiatric Clinics of North America, 14(1), 83-104. http://dx.doi.org/10.1016 /j.chc.2004.07.002

Gruzelier, J. H. (2014). EEG-neurofeedback for optimising performance. I: A review of cognitive and affective outcome in healthy participants. Neuroscience and Biobehavioral Reviews, 44, 124-141. http://dx.doi.org/10.1016 /j.neubiorev.2013.09.015

Hammond, D. C. (2005). Neurofeedback treatment of depression and anxiety. Journal of Adult Development, 12(2), 131-137. http://dx.doi.org/10.1007/s10804-005-7029-5

Hill, E. L., \& Frith, U. (2003). Understanding autism: Insights from mind and brain. Philosophical Transactions of the Royal Society of London B: Biological Sciences, 358(1430), 281289. http://dx.doi.org/10.1098/rstb.2002.1209

Hoedlmoser, K., Pecherstorfer, T., Gruber, G., Anderer, P., Doppelmayr, M., Klimesch, W., \& Schabus, M. (2008). Instrumental conditioning of human sensorimotor rhythm (12$15 \mathrm{~Hz})$ and its impact on sleep as well as declarative learning. Sleep, 31(10), 1401-1408.

Holtmann, M., Bölte, S., \& Poustka, F. (2007). Autism spectrum disorders: Sex differences in autistic behaviour domains and coexisting psychopathology. Developmental Medicine and Child Neurology, 49(5), 361-366. http://dx.doi.org /10.1111/j.1469-8749.2007.00361.x

Holtmann, M., Steiner, S., Hohmann, S., Poustka, L., Banaschewski, T., \& Bölte, S. (2011). Neurofeedback in autism spectrum disorders. Developmental Medicine and Child Neurology, 53(11), 986-993. http://dx.doi.org /10.1111/j.1469-8749.2011.04043.x

Keller, I., \& Garbacenkaite, R. (2015). Neurofeedback in Three Patients in the State of Unresponsive Wakefulness. Applied Psychophysiology and Biofeedback, 40(4), 349-356. http://dx.doi.org/10.1007/s10484-015-9296-7 
Kouijzer, M. E. J., de Moor, J. M. H., Gerrits, B. J. L., Buitelaar, J. K., \& van Schie, H. T. (2009). Long-term effects of neurofeedback treatment in autism. Research in Autism Spectrum Disorders, 3(2), 496-501. http://dx.doi.org/10.1016 /j.rasd.2008.10.003

Kouijzer, M. E. J., de Moor, J. M. H., Gerrits, B. J. L., Congedo, M., \& van Schie, H. T. (2009). Neurofeedback improves executive functioning in children with autism spectrum disorders. Research in Autism Spectrum Disorders, 3(1), 145-162. http://dx.doi.org/10.1016/j.rasd.2008.05.001

Lubar, J. F. (1997). Neocortical dynamics: Implications for understanding the role of neurofeedback and related techniques for the enhancement of attention. Applied Psychophysiology and Biofeedback, 22(2), 111-126.

Marshall, L., Mölle, M., Hallschmid, M., \& Born, J. (2004). Transcranial direct current stimulation during sleep improves declarative memory. The Journal of Neuroscience, 24(44), 9985-9992. http://dx.doi.org/10.1523/JNEUROSCI.272504.2004

Rogel, A., Guez, J., Getter, N., Keha, E., Cohen, T., Amor, T., \& Todder, D. (2015). Transient Adverse Side Effects During Neurofeedback Training: A Randomized, Sham-Controlled, Double Blind Study. Applied Psychophysiology and Biofeedback, 40(3), 209-218. http://dx.doi.org/10.1007 /s10484-015-9289-6
Sajith, S. G., \& Clarke, D. (2007). Melatonin and sleep disorders associated with intellectual disability: A clinical review. Journal of Intellectual Disability Research, 51(1), 2-13. http://dx.doi.org/10.1111/j.1365-2788.2006.00893.x

Sterman, M. B. (2000). Basic concepts and clinical findings in the treatment of seizure disorders with EEG operant conditioning. Clinical EEG and Neuroscience, 31(1), 45-55. http://dx.doi.org/10.1177/155005940003100111

Sterman, M. B., \& Bowersox, S. S. (1981). Sensorimotor electroencephalogram rhythmic activity: a functional gate mechanism. Sleep, 4(4), 408-422.

Sterman, M. B., \& Egner, T. (2006). Foundation and practice of neurofeedback for the treatment of epilepsy. Applied Psychophysiology and Biofeedback, 31(1), 21-35. http://dx.doi.org/10.1007/s10484-006-9002-x

Tononi, G., \& Cirelli, C. (2006). Sleep function and synaptic homeostasis. Sleep Medicine Reviews, 10(1), 49-62. http://dx.doi.org/10.1016/j.smrv.2005.05.002

Tuchman, R. (2000). Treatment of seizure disorders and EEG abnormalities in children with autism spectrum disorders. Journal of Autism and Developmental Disorders, 30(5), 485489. http://dx.doi.org/10.1023/A:1005572128200

Received: June 29, 2016

Accepted: August 14, 2016

Published: September 2, 2016 